\title{
Detector and data characterization at GEO 600
}

\author{
M Hewitson (for the LIGO Scientific Collaboration)
}

Max-Planck-Institut für Gravitationsphysik (Albert-Einstein-Institut) und Universität Hannover, Außenstelle Hannover, Callinstr. 38, 30167 Hannover, Germany

E-mail: martin.hewitson@aei.mpg.de

Received 23 April 2007, in final form 3 June 2007

Published 19 September 2007

Online at stacks.iop.org/CQG/24/S445

\begin{abstract}
GEO 600 is one of a few detectors in the world searching for gravitational wave signals from various astronomical sources. The commissioning and characterization of gravitational wave detectors is a highly complex activity that requires the use of many sophisticated analysis procedures. In order to extract all possible astronomical information from the data recorded at GEO 600 , the detector and the data must be understood and characterized as well as possible so that the data can make the largest possible contribution in any subsequent analyses. This paper provides a review of some of the aspects of detector and data characterization that takes place at GEO 600.
\end{abstract}

PACS numbers: 95.55.Ym, 04.80.Nn

(Some figures in this article are in colour only in the electronic version)

\section{Introduction}

Laser-interferometric gravitational wave $(\mathrm{GW})$ detectors are highly complex instruments designed to detect differential length changes of the order 1 part in $10^{20}$ at frequencies between $50 \mathrm{~Hz}$ and a few $\mathrm{kHz}$. The construction and commissioning of such instruments takes many years. Towards the end of the commissioning phase, increasingly more emphasis is placed on characterizing both the instrument itself and the data it produces so as to allow for the best interpretation of the data during subsequent analyses.

Such gravitational wave detectors as those described in [1-4] record signals from many subsystems. Typically hundreds of channels are recorded with sample rates ranging from a few $\mathrm{Hz}$ to a few $\mathrm{kHz}$.

GEO 600 is one such laser interferometric GW detector situated near Hannover, Germany. A schematic layout of GEO 600 is given in figure 1. The sensitivity of GEO 600 is typically limited by a complicated tapestry of environmental and instrumental noise sources which couple by varying degrees to the main gravitational wave channel. In the early stages of commissioning it was possible to find limiting noise sources whose removal would bring 


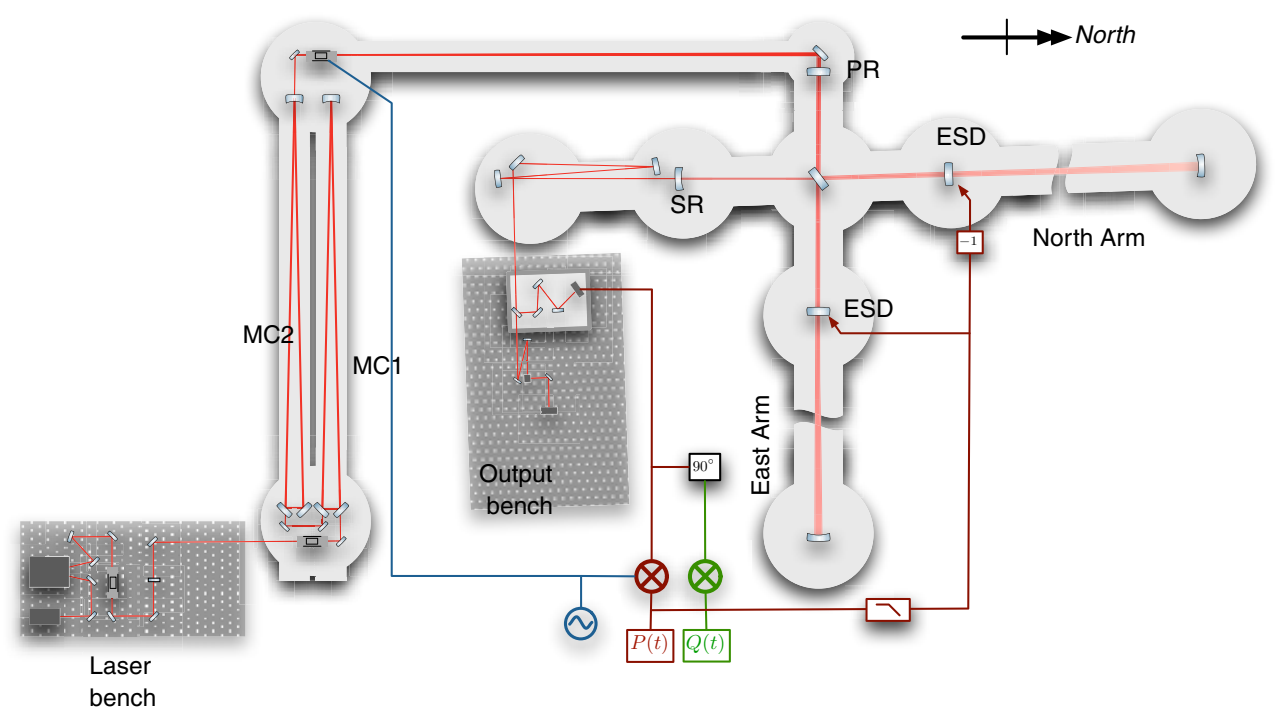

Figure 1. Schematic layout of GEO 600 showing the main longitudinal control scheme. The light leaving the laser enters two sequential mode-cleaners. Control side-bands are then applied to the light before it enters the power-recycling cavity (PR). The side-band light (and any residual carrier light) leaves the Michelson at the south port and is then resonantly enhanced by the signal-recycling cavity (SR). An output telescope then focuses and steers the light onto the output bench where it is detected on a photodiode.

large increases in sensitivity (factors of 2 and more). As the commissioning proceeds and the sensitivity of the instrument increases, the limiting noise sources tend to, more and more, overlap, such that the removal of any one brings only a small (few per cent) increase in sensitivity. It is therefore essential to track, with care, the current sensitivity of the instrument so that any improvement can be recognized and associated with a particular experiment or hardware change.

In order to identify changes in sensitivity, it is first useful to have a reliable and accurate calibration of the main gravitational wave channel. This is especially true in GEO 600 since, due to the signal-recycling cavity, gravitational wave signals will be spread between two main output signals in a frequency- and time-dependent way. (These two output signals are referred to as $P$ and $Q$ in the remainder of this paper.) In order to recover the best sensitivity from the instrument the calibration scheme has to combine these two output signals into one, optimal gravitational wave signal. This makes recognizing sensitivity improvements much simpler, since only one signal has to be monitored and tracked.

\section{Detector characterization at GEO 600}

In GEO 600, tracking of the instrument sensitivity is primarily done using 'summary reports'. These reports are generated automatically three times per day and are of fixed format so as to allow quick comparison between one report and another. The contents of each summary report include

- noise-spectral densities of the two main calibrated output channels and the optimally combined GW channel; 


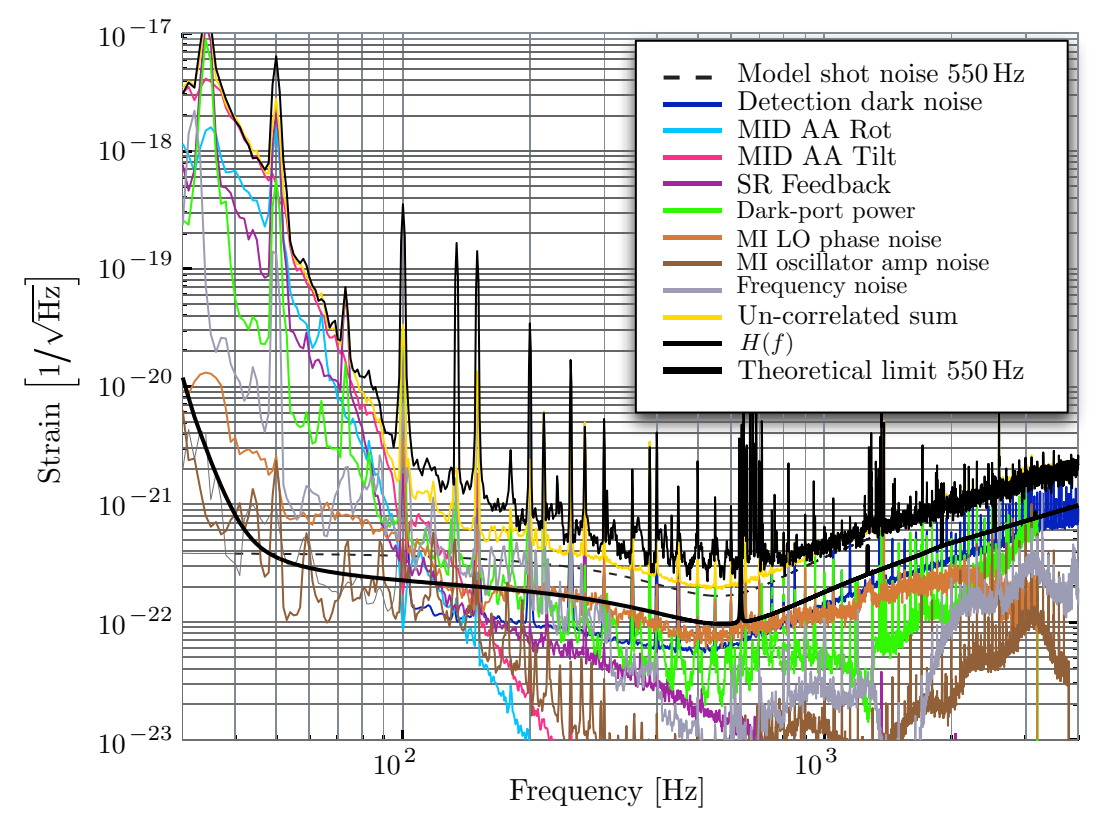

Figure 2. GEO 600 noise budget for September 2006.

- sensitivity to detect some standard inspiral and burst waveforms;

- basic data quality information such as lock status, maintenance mode, etc;

- time evolution of the detector model parameters derived during the calibration routine;

- glitch rates (from mHACR burst detection algorithm; see section 3.1) of the main GW channel as well as many auxiliary channels, and

- reported saturations of data acquisition channels.

Summary reports provide a quick overview of a long stretch of data ( $8 \mathrm{~h}$, in this case). This allows the stationarity, glitchiness and calibration quality of a data segment to be assessed at a glance. In addition to information gleaned from the analysis of the main GW channel alone, online coincidence tests of transient events detected in multiple auxiliary channels with those detected in the main GW channel are performed. By doing this, it is possible to quickly identify if a particular auxiliary channel is potentially responsible for a subset of the glitches detected in the main GW channel. If the coincidence of events appears non-negligible, a follow up analysis is typically performed and an attempt is made, either to mitigate the source of the glitches, or to create a veto from the auxiliary channel.

\subsection{Noise projections}

The advanced stages of commissioning a GW detector rely on a good understanding of the noise in the main GW channel in order that the limiting noise sources can be identified and removed or reduced. The noise spectrum of the main GW channel comprises noise from many subsystems within the interferometer. Identifying the limiting noise sources and determining how much each contributes to the total noise observed in the main $\mathrm{GW}$ channel is a process termed 'noise projections' [5]. The result of a set of noise projections is a 'noise budget'. A typical noise budget for GEO 600 from September 2006 is shown in figure 2. At this time, 
the noise present in the main GW channel of GEO 600 is reasonably well understood, but still some unknown noise is present as can be seen from the discrepancy between the $H(f)$ trace and the uncorrelated sum of all the identified noise sources (yellow trace).

\subsection{Calibration of the main gravitational wave channel}

A lot of the detector and data characterization performed at GEO 600 relies upon a prompt and optimal calibration of the detector. GEO 600 uses an online, time-domain calibration routine which is described in detail in [6-9]. Briefly, a model of the detector response is fit to a set of measurements made every second. The measurements are made at discrete frequencies by injecting 'calibration lines' into the differential length control servo. The model comprises two actuator transfer functions (one for the electrostatic drive actuator, one for the intermediate-mass coil/magnet actuator [10]) and two variable transfer functions which describe the response of the Michelson interferometer (MI) to differential arm-length changes. (Due to the signal-recycling cavity and the heterodyne readout scheme used at GEO 600, GW information can be obtained by demodulating the MI radio frequency control sidebands detected at the main output photodiode in two quadratures.) These transfer functions are usually termed 'optical gain'. One of the demodulated outputs serves as the error point for the longitudinal control servo of the Michelson interferometer. Together these two transfer functions have eight free parameters.

An optimization routine is used to obtain estimates of these eight parameters for each second of data that is collected. The parameter estimates are then low-pass filtered so as to reduce measurement noise (due to limited signal-to-noise ratio (SNR) of injected calibration lines); this means that the calibration routine is capable of tracking fluctuations in the instrument response on time scales of about $10 \mathrm{~s}$ and slower. These parametrized optical responses are inverted and converted into digital time-domain filters which then filter the error-point signals to create two estimates of the apparent differential length change of the Michelson. Since these error-point signals are derived from sensing inside a control-loop (in-loop), we also need to correct for the loop-gain of the servo. This is achieved by adding filtered versions (through actuator transfer functions) of the measured feedback signals to the two differential length change estimates. Scaling the two differential displacement signals by the length of the arms yields two estimates of the detected strain of GEO 600. These two estimates are then combined in two ways: one way to produce an optimal strain data stream (with optimal SNR for GW signals) [11], and another way (difference) to produce a null-stream (a data stream containing negligible GWs) [12].

2.2.1. Absolute calibration accuracy. Accurate calibration of GW detectors not only serves to guide the commissioning process, but is also essential for multi-detector analyses. This is particularly the case when any coherent analysis is performed (for example, when searching for pulsar signals $[13,14]$ ) where phase inaccuracies could potentially destroy a signal. In addition, a lot of research effort is currently focussing on the use of single- and multidetector null-stream constructions [15-17]. Null-streams, as the name suggests, combine the output(s) of single or multiple detectors in order to produce a data stream that contains no GW information. A simple example of such a null-stream can be constructed from the calibrated strain signals from the two collocated LIGO Hanford detectors; there the null-stream is simply the difference of the two strain signals. However, it is clear that if the two strain signals are subject to systematic calibration errors then the resulting null-stream will not be truly null and could contain residual energy from any detected GW signal. This can significantly reduce the veto power of the null-stream [18]. 


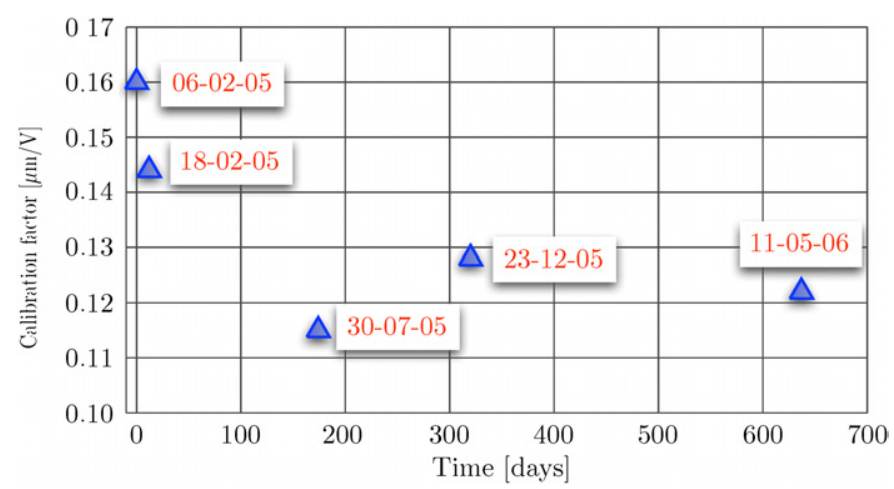

Figure 3. A plot of the measured calibration factor for the electrostatic drives over time. The measured calibration factor is scaled to be the dc gain used in the model of the actuator transfer function. This is derived for a complex pole at $0.8 \mathrm{~Hz}$ with a $Q$ of 2 .

The absolute calibration at GEO 600 relies on determining the strength of the electrostatic drive actuators (ESDs), that is, determining how much the test mass is displaced for a given drive signal. The procedure of calibrating the ESDs is a complicated one and ultimately sets the accuracy with which the detector can be calibrated. The ESD calibration procedure is summarized as follows.

- The strength of the two ESDs (north and east) is balanced by moving the reaction pendulum chain of one closer or further away from the test mass until both drives give the same displacement for a given drive signal. This is done with the detector locked. The drive signal is typically injected above $1 \mathrm{kHz}$ so as to be well outside the bandwidth of the MI longitudinal servo, and the typical adjustment required is of the order of a few per cent.

- The length of the first suspended mode-cleaner is taken as the standard to which we calibrate. This length is known very accurately $(\ll 1 \%)$ and yields a free-spectral range of around $37.465 \mathrm{MHz}$. From this, we can calibrate the piezo used to change the frequency of the master laser crystal. We do this by driving the piezo in its linear range to produce frequency sweeps that span multiple FSRs of the first mode-cleaner. By measuring the voltage needed to drive the master laser over one FSR we can get a calibration of the piezo.

- The feedback signal of the frequency stabilization loop is applied to the length of the second mode-cleaner so the next step is to calibrate that actuator back to the feedback that is applied to the master laser piezo (which is responsible for shifting the laser frequency so that the power-recycling cavity remains resonant).

- By driving the east ESD alone we produce a certain amount of common mode displacement of the Michelson. Thus we can calibrate the strength of the east ESD against the feedback signal of the frequency stabilization servo.

This whole procedure yields a calibration of the east ESD to around $\pm 5 \%$ accuracy. (The north ESD is the same since we balanced them at the beginning of the procedure.) Figure 3 shows measurements of the calibration factor made at various times over a period of about 18 months. This calibration procedure is time consuming and as such is typically carried out at the beginning and end of science runs, or when a hardware change is known to affect this value. The changes in the calibration constant can usually be accounted for by either a venting of the vacuum system which can lead to changes in the spacing of the ESDs from 

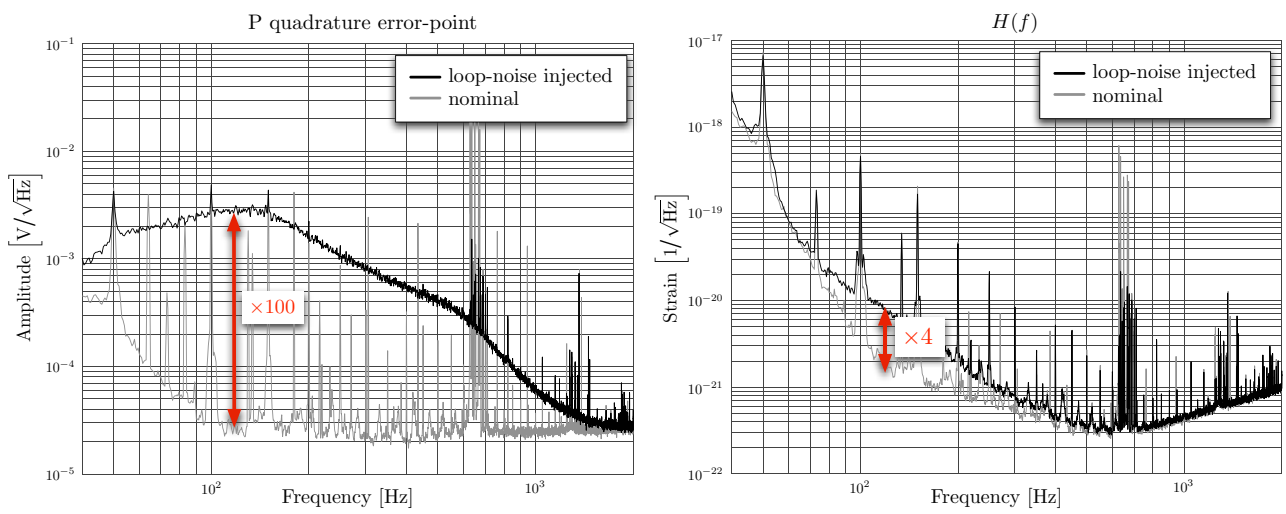

Figure 4. Left: spectral density estimates of the $P$ quadrature error-point signal for nominal (light trace) conditions and when noise is injected in the loop between the error point and feedback point (dark trace). Right: spectral density estimates of the main reconstructed strain signal for the same two cases as described above.

their respective reaction masses, or due to changes to electronics after the point where the calibration is taken.

2.2.2. Relative calibration accuracy. Having an accurate absolute (dc) calibration is only part of the picture. It is also important that the time-domain $h(t)$ calibration routine does not introduce any (large) frequency-dependent systematic errors. Such errors can arise from inaccuracies in any digital filters designed to mimic the response of the actuators, or in the combining process, or due to optimization routine which ultimately yields the digital filters which invert the optical response of the detector.

The calibration accuracy relative to the absolute (dc) calibration can be assessed in a number of ways. Since the calibration routine uses both the error points and feedback signals of the Michelson length control servo to derive estimates of the detected strain, any noise that is added between the measurement points of the error-point and feedback signals will be automatically suppressed in the final calibrated data stream. How good this suppression is depends on the relative calibration accuracy. If the calibration routine would do a perfect job, then noise added in this way would be completely removed from the final $h(t)$ signal. We can test this by injecting white noise between the error point and feedback points of the servo such that the injected noise dominates the nominal noise in the $P$ quadrature. The left plot of figure 4 shows spectral densities of the $P$ quadrature error point for two cases: the lighter trace represents nominal operating conditions of the detector; the other, darker trace is for a time where noise is injected into the servo. We can see that we dominate the nominal noise spectrum by about a factor of 100 at $100 \mathrm{~Hz}$. The right plot of figure 4 shows spectral density estimates for the main $h(t)$ signal for the same two cases. Here we can see that the injected noise is suppressed to an extent that it appear to dominate the nominal noise floor by about a factor of 4 around $100 \mathrm{~Hz}$. For this suppression of around a factor 25 to happen, the relative calibration accuracy must be within a few per cent in magnitude and a few degrees in phase.

A clearer test is to drive the ESDs with white noise, this time injecting the noise after the measurement point of the feedback signal (so that the additional noise is not subtracted in the calibration process), and compare what we get in $h(t)$ to what we expect from the model of the electrostatic drive. The model of ESDs is based on measurements made in the 

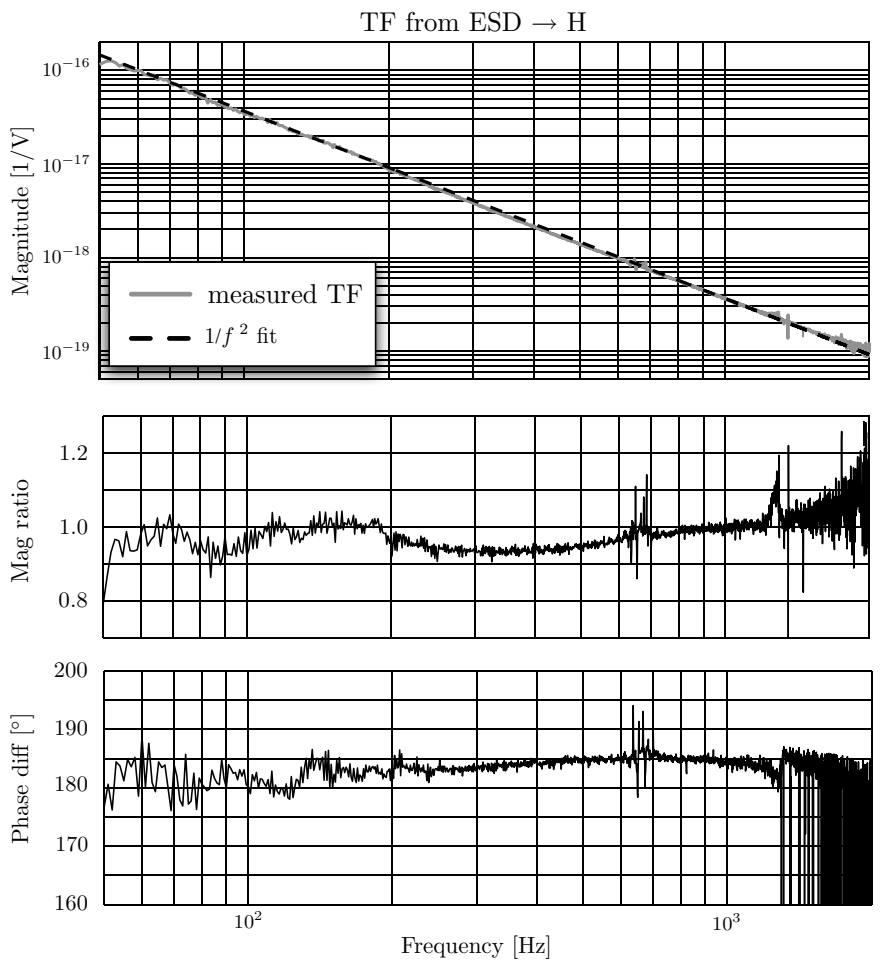

Figure 5. Measured and modelled transfer functions from the input to the ESDs to the main calibrated output of GEO 600. The middle and lower traces show the magnitude ratio and phase difference respectively.

power-recycled Michelson mode of GEO 600. In this mode, the optical response of the detector to differential length changes is known to be flat in frequency. Therefore, by driving the ESDs with white noise, we can directly look in the Michelson error signal and measure the transfer function of the ESDs from a voltage signal input to something proportional to the induced displacement. Using this knowledge of the frequency response and the absolute calibration described above, we get an accurate model of the ESD response.

The upper plot of figure 5 shows a measured transfer function from ESD input to the main calibrated output of GEO 600. Also shown is a model $1 / f^{2}$ response which is a very good approximation to the ESD response below $2 \mathrm{kHz}$. The middle and lower plot of figure 5 shows the amplitude ratio and phase difference between the measured and model responses of the transfer function. From this, we deduce that the relative calibration accuracy of the GEO 600 calibration process is within $10 \%$ and $10^{\circ}$ across the detection band.

\section{Data characterization}

Data characterization in GEO 600 focuses mainly on the development of possible veto methods which can then be used to reduce the candidate glitch events detected in the main detector output. This focus arises from the fact that the sensitivity of GEO 600 comes closest to the larger scale LIGO detectors at frequencies above $400 \mathrm{~Hz}$. At these frequencies, the most promising scientific contribution that data from GEO 600 can make is in the search for 


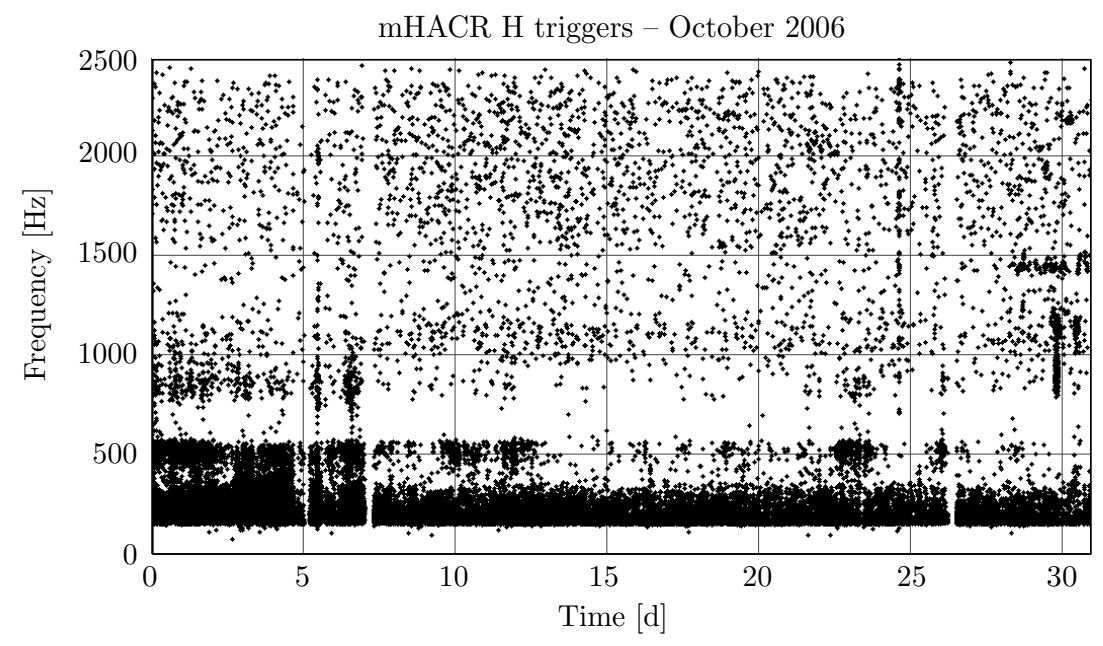

Figure 6. A time-frequency map of mHACR triggers detected in $h(t)$ during the month of October 2006.

unmodelled transient bursts of GW energy. The following sections look at the detection of transient signals in the recorded channels of GEO 600, as well as at the primary data quality flags and veto channel used for the reduction of candidate burst events.

\subsection{Detecting transient events}

The HACR algorithm [19, 20] uses a time-frequency description of a data segment to identify transient events (or triggers). A modified version of the algorithm, mHACR, which has improved parameter estimation, is used in all of the transient signal studies shown in this paper. A description of the mHACR algorithm and, in particular, a discussion of the parametrization of identified events, are given in [21].

\subsection{Vetoing transient events detected in the main gravitational wave channel}

The mHACR algorithm is routinely run over many output channels of GEO 600. Figure 6 shows a time-frequency map of events detected in the main GW channel by the mHACR algorithm for the entire month of October 2006. The events from this analysis are stored in a database which is then mined to look for coincident events between auxiliary channels and the main $h(t)$ channel. Preliminary coincident analysis is done in an automated way as part of the 'summary reports'. When significant coincidences are identified between a particular auxiliary channel and the main GW channel, a suitable veto method is developed in order to produce a list of veto intervals which can be applied to the event list for the main GW channel. Suitable veto methods include simple statistical vetoes, statistical vetoes with additional constraints, for example, with amplitude consistency or multiple coincidence windows, and vetoes that use known instrumental couplings.

3.2.1. Null-stream veto. One special veto method that is available in GEO 600 is the socalled GEO null-stream veto. The null-stream is constructed by taking the difference of the two calibrated output quadratures of GEO 600 to yield a data stream that contains practically no 


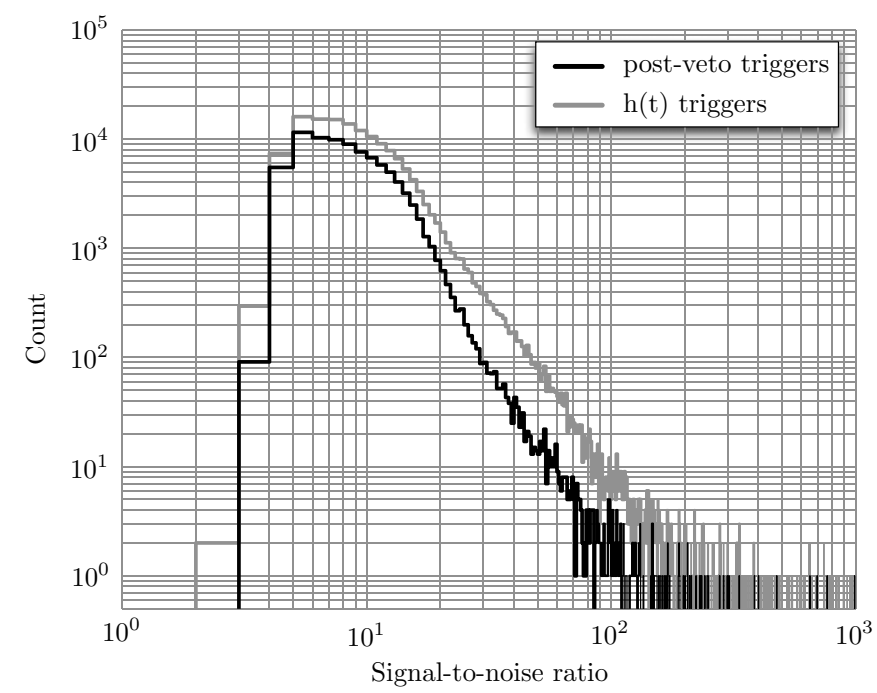

Figure 7. Histogram of the SNR of events detected in the main GW channel of GEO 600 before and after vetoing with the null-stream signal.

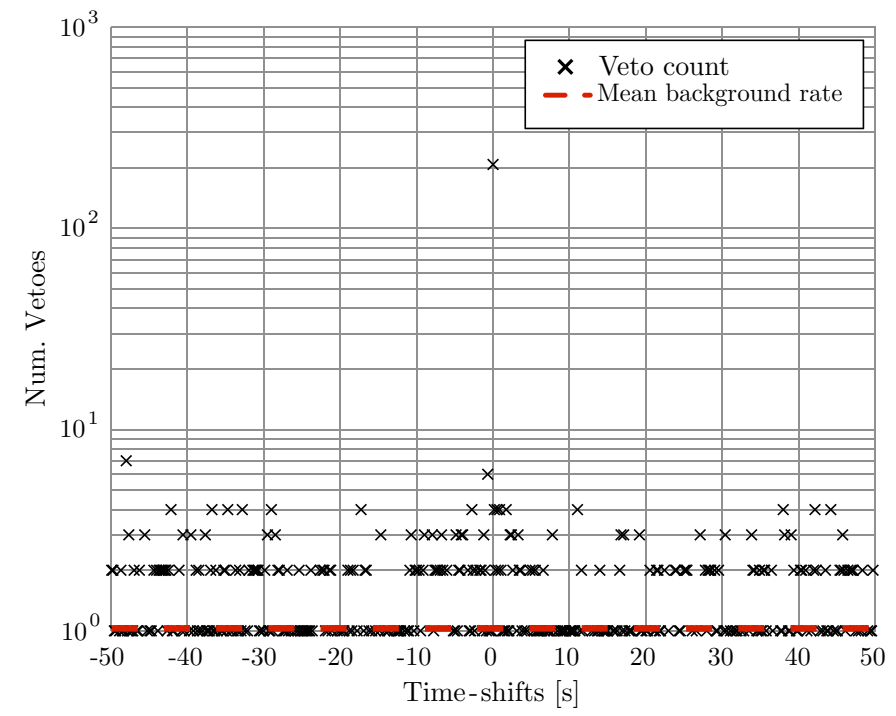

Figure 8. Time-shift analysis of the events from the first day of September 2006. The background coincidence rate is about 1 per day.

GW information. This method looks for coincident events between the null-stream output of GEO 600 and the main GW channel. We demand time, frequency and amplitude consistency when looking for coincident events. This veto method is described in more detail in [12].

Applying this method to the events detected in September 2006 yields a significant reduction in the candidate event list of the main GW channel. Figure 7 shows histograms of the SNR of events detected in the main GW channel before and after applying the null-stream veto. We can see that a large fraction of the high SNR events is vetoed by this method. 
Figure 8 shows the result of a time-shift analysis performed on this one day of events from the beginning of September 2006. A time-offset is added to the time-stamp of events detected in the null-stream and the coincidence analysis is then performed. Repeating this for many different time-offsets yields a measure of the accidental coincidence rate that exists between the main GW channel and the null-stream. We can see that for a time-shift of zero, we veto around 200 events per day, whereas, for all other time-shifts we veto, on average, one event per day.

\section{Summary}

The detector and data characterization carried out at GEO 600 is now at a mature stage with glitch and sensitivity studies being carried out continuously on all recorded data. The complicated optical configuration together with the heterodyne readout scheme used at GEO 600 means that a continuous and online calibration of the main output data streams is necessary to interpret the sensitivity of the detector and hence to monitor and assess the impact of any particular commissioning step.

This paper presents an overview of some of the typical characterization tasks that are performed at GEO 600. In particular, the calibration of the main GW output of GEO 600 is reviewed and the accuracy of the process is discussed. We show that the calibration of GEO 600 is accurate to about $10 \%$ between $50 \mathrm{~Hz}$ and $2 \mathrm{kHz}$.

We also present the results of some veto analyses performed on data taken from the S5 science run, focussing in particular on the use of the null-stream veto.

\section{Acknowledgments}

The authors are grateful for support from PPARC and the University of Glasgow in the UK, and the BMBF and the state of Lower Saxony in Germany. This document has been assigned LIGO Laboratory document number LIGO-P070035-00-Z.

\section{References}

[1] Hild S (for the LIGO Scientific Collaboration) 2006 The status of GEO 600 Class. Quantum Grav. 23 S643-51

[2] Waldman S J (for the LIGO Science Collaboration) 2006 Status of LIGO at the start of the fifth science run Class. Quantum Grav. 23 S653-60

[3] Acernese F et al 2006 The Virgo status Class. Quantum Grav. 23 S635-42

[4] Ando M (the TAMA Collaboration) 2005 Current status of the TAMA300 gravitational-wave detector Class. Quantum Grav. 22 S881-9

[5] Smith J R, Ajith P, Grote H, Hewitson M, Hild S, Lück H, Strain K A, Willke B, Hough J and Danzmann K 2006 Linear projection of technical noise for interferometric gravitational-wave detectors Class. Quantum Grav. 23 527-37

[6] Hewitson M, Grote H, Heinzel G, Strain K A, Ward H and Weiland U 2003 Calibration of the power-recycled gravitational wave detector, GEO 600 Rev. Sci. Instrum. 744184

[7] Hewitson M, Grote H, Heinzel G, Strain K A, Ward H and Weiland U 2003 Calibration of GEO 600 for the S1 science run Class. Quantum Grav. 20 S885-93

[8] Hewitson M, Heinzel G, Smith J R, Strain K A and Ward H 2004 Principles of calibrating the dual-recycled GEO 600 Rev. Sci. Instrum. $\mathbf{7 5} 4702$

[9] Hewitson Met al 2004 Calibration of the dual-recycled GEO 600 detector for the S3 science run Class. Quantum Grav. 21 S1711-22

[10] Gossler S et al 2002 The modecleaner system and suspension aspects of GEO 600 Class. Quantum Grav. $191835-42$ 
[11] Hewitson M, Grote H, Hild S, Lück H, Ajith P, Smith J R, Strain K A, Willke B and Woan G 2005 Optimal time-domain combination of the two calibrated output quadratures of GEO 600 Class. Quantum Grav. 22 4253-61

[12] Hewitson M and Ajith P 2005 Using the null-stream of GEO 600 to veto transient events in the detector output Class. Quantum Grav. 22 4903-12

[13] Dupuis R J and Woan G 2005 Bayesian estimation of pulsar parameters from gravitational wave data Phys. Rev. D 72102002

[14] LIGO Scientific Collaboration 2004 Upper limits on the strength of periodic gravitational waves from PSR J1939+2134 Class. Quantum Grav. 21 S671-6

[15] Gürsel Y and Tinto M 1989 Near optimal solution to the inverse problem for gravitational-wave bursts Phys. Rev. D 403884

[16] Wen L and Schutz B F 2005 Coherent network detection of gravitational waves: the redundancy veto Class. Quantum Grav. 22 S1321-35

[17] Chatterji S, Lazzarini A, Stein L, Sutton P, Searle A and Tinto M 2006 Coherent network analysis technique for discriminating gravitational-wave bursts from instrumental noise Phys. Rev. D 74082005

[18] Ajith P, Hewitson M and Heng I S 2006 Null-stream veto for two co-located detectors: implementation issues Class. Quantum Grav. 23 S741-9

[19] Balasubramanian R, Grote H, Heng I S, Hewitson M, Lück H, Smith J R, Strain K A, Ward H and Willke B 2005 Results from the first burst hardware injections performed on GEO 600 Class. Quantum Grav. 22 3015-28

[20] Heng I S, Balasubramanian R, Sathyaprakash B S and Schutz B F 2004 First steps towards characterizing the hierarchical algorithm for curves and ridges pipeline Class. Quantum Grav. 21 S821-6

[21] Hild S, Ajith P and Hewitson M 2007 A statistical veto method employing an amplitude consistency check Class. Quantum Grav. submitted 